\title{
Finite Element Analysis of Bend Test of Sandwich Structures Using Strain Energy Based Homogenization Method
}

\author{
Hassan Ijaz, ${ }^{1}$ Waqas Saleem, ${ }^{1}$ Muhammad Zain-ul-Abdein, ${ }^{1}$ Tarek Mabrouki, ${ }^{2}$ \\ Saeed Rubaiee, ${ }^{3}$ and Abdullah Salmeen Bin Mahfouz ${ }^{4}$ \\ ${ }^{1}$ Mechanical Engineering Department, University of Jeddah, Jeddah, Saudi Arabia \\ ${ }^{2}$ Mechanical Engineering Department, University of Tunis El Manar, ENIT, Tunis, Tunisia \\ ${ }^{3}$ Industrial Engineering Department, University of Jeddah, Jeddah, Saudi Arabia \\ ${ }^{4}$ Chemical Engineering Department, University of Jeddah, Jeddah, Saudi Arabia \\ Correspondence should be addressed to Hassan Ijaz; hassan605@yahoo.com
}

Received 23 February 2017; Revised 11 April 2017; Accepted 20 April 2017; Published 11 June 2017

Academic Editor: Frederic Dumur

Copyright (C) 2017 Hassan Ijaz et al. This is an open access article distributed under the Creative Commons Attribution License, which permits unrestricted use, distribution, and reproduction in any medium, provided the original work is properly cited.

\begin{abstract}
The purpose of this article is to present a simplified methodology for analysis of sandwich structures using the homogenization method. This methodology is based upon the strain energy criterion. Normally, sandwich structures are composed of hexagonal core and face sheets and a complete and complex hexagonal core is modeled for finite element (FE) structural analysis. In the present work, the hexagonal core is replaced by a simple equivalent volume for FE analysis. The properties of an equivalent volume were calculated by taking a single representative cell for the entire core structure and the analysis was performed to determine the effective elastic orthotropic modulus of the equivalent volume. Since each elemental cell of the hexagonal core repeats itself within the in-plane direction, periodic boundary conditions were applied to the single cell to obtain the more realistic values of effective modulus. A sandwich beam was then modeled using determined effective properties. 3D FE analysis of Three-and Four-Point Bend Tests (3PBT and 4PBT) for sandwich structures having an equivalent polypropylene honeycomb core and Glass Fiber Reinforced Plastic (GFRP) composite face sheets are performed in the present study. The authenticity of the proposed methodology has been verified by comparing the simulation results with the experimental bend test results on hexagonal core sandwich beams.
\end{abstract}

\section{Introduction}

Applications of composite sandwich structures are continuously increasing in the recent times due to their excellent out-of-plane shear and compressive properties. Modern manufacturing aerospace, ship building, and automotive industries are the main users of sandwich structures. A typical sandwich structure consists of a central core material covered by top and bottom face sheets. Generally honeycomb, truss, and foam are used as the inner cores in sandwich structures. Nomex, Polypropylene, and Aluminium are regularly used core materials, whereas, Glass Fiber Reinforced Plastic (GFRP), Carbon Fiber Reinforced Plastic (CFRP), and Aluminium are commonly used face sheet materials.

Composite sandwiches are used as substantial structures in cars, ships, beams, and so forth. Complex and large hexagonal honeycomb core shapes are not only difficult to model but also are computationally expensive. Due to these limitations, an alternative strategy is desired in which a complex shaped core material may be replaced by a simple equivalent volume having elastic orthotropic properties.

Many authors attempted to determine the equivalent properties of central core by separately modeling the honeycomb core sheet with hexagonal unit cells [1-6]. Each cell of the honeycomb sheet periodically repeats itself within inplane direction. The required equivalent core properties are the in-plane $\left(\mathrm{E}_{11}, \mathrm{E}_{22}\right)$ and out-of-plane $\left(\mathrm{E}_{33}\right)$ elastic moduli, in-plane $\left(G_{12}\right)$ and out-of-plane $\left(G_{13}, G_{23}\right)$ shear moduli, and the Poisson ratios $\left(v_{12}, v_{13}\right.$, and $\left.v_{23}\right)$. The homogenization method is based on the mechanical equivalence between the microstructures of RVE (representative volume element) and a similar homogeneous macroscopic volume. 


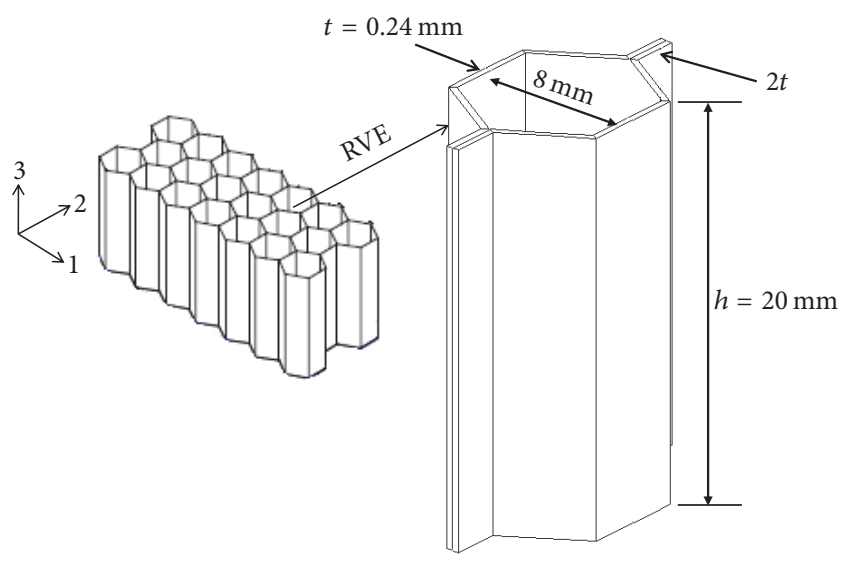

FIgURE 1: Representative volume element (RVE).

Different authors like Kelsey et al. [7], Gibson and Ashby [8], Zhang and Ashby [9], Klintworth and Stronge [10], Hohe and Becker [11], and Yang and Becker [12] developed the analytical schemes to estimate the equivalent properties of cellular core. Normally analytical theories are shape dependent and also not able to predict all the 9 engineering constants of the elastic orthotropic properties. In recent years, finite element analysis (FEA) has become a popular tool to determine the equivalent elastic properties of any cellular structure of periodic nature [13-17].

In the present study only a single hexagonal element with periodic boundary conditions has been modeled to determine the equivalent properties. This approach to determine the equivalent properties is based on the work of Gornet et al. $[13,15]$. The required orthotropic properties in terms of 9 engineering constants are determined using the strain energy based homogenization method. Subsequently, a 3D sandwich structure with face sheets and simple inner core volume was modeled along with the calculated equivalent properties and analyzed for Three-Point Bend Test (3PBT) and Four-Point Bend Test (4PBT). For the 3PBT and 4PBT finite element (FE) simulations, a complex hexagonal honeycomb is replaced by the equivalent $3 \mathrm{D}$ core material. Finally, the results were compared with the experimental data.

This article is organized as follows: representative volume element (RVE) for honeycomb core material is defined and described in Section 2. Mathematical formulation is explained in Section 3. FEA results and its comparison with experimental results are discussed in Section 4. Finally, some concluding remarks are made in Section 5.

\section{Representative Volume Element (RVE)}

Figure 1 shows a representative volume element (RVE) for overexpanded hexagonal honeycomb material along with necessary orthotropic basis, that is, axes 1, 2, and 3. Now by successive in-plane translation of RVEs, one can obtain a larger volume of the honeycomb cellular structure. RVE for honeycomb core material and corresponding equivalent homogenized volume is shown in Figure 2. Once the orthotropic properties of homogenized volume are known, one can model the large and complex sandwich structures with simple homogenized volume for different FE analyses.

\section{Formulation}

By considering $Y$ to be the RVE and $|Y|$ its volume measurement, the average of the second-order tensor $X_{I J}$ may be represented by the following relation:

$$
\left\langle X_{I J}\right\rangle=\frac{1}{|Y|} \int_{Y} X_{I J}(Y) d Y .
$$

One can express the average stress and strain tensors as $\Sigma_{I J}=\left\langle\sigma_{I J}\right\rangle$ and $E_{I J}=\left\langle\varepsilon_{I J}\right\rangle$, respectively. Periodic homogenization is based upon the data average over the strain field and then one determines the compliance operator $C^{\mathrm{RVE}}$ of the RVE by imposing displacement boundary conditions [13]. Here, Hill-Mandel [18, 19] energy approach is used to calculate the equivalent homogenized components of $6 \times 6$ compliance matrix $C_{I J}^{\mathrm{RVE}}$. Now homogenized stress and strain tensors are related to each other with the help of the following relation:

$$
\begin{aligned}
& \Sigma=C^{\mathrm{RVE}} E \\
& E=S^{\mathrm{RVE}} \Sigma .
\end{aligned}
$$

Here $S^{\mathrm{RVE}}$ represents the $6 \times 6$ stiffness matrix. The homogenized mechanical stiffness characteristics $\left(C^{\mathrm{RVE}}\right)$ of the RVE can be derived with the Hill-Mandel theorem [18, 19]. The Hill-Mandel theorem equates the RVE energy to the average of the energies of its components:

$$
\varepsilon_{I} C_{I J}^{\mathrm{RVE}} \varepsilon_{J}=\frac{1}{|Y|^{\mathrm{RVE}}} \int_{Y} \operatorname{Tr}[\sigma \varepsilon] d Y .
$$

The 21 components of the orthotropic compliance matrix $C_{I J}^{\mathrm{RVE}}$ can be determined by applying 21 linear combinations of applied loading on RVE and the resultant set of the corresponding equations may be expressed as follows [13, 15]:

$$
\begin{aligned}
& \varepsilon_{I} C_{I I}^{\mathrm{RVE}} \varepsilon_{I}=\frac{1}{|Y|^{\mathrm{RVE}}} \int_{Y} \operatorname{Tr}[\sigma \varepsilon] d Y \quad I \in[1,3] \\
& \sqrt{2} \varepsilon_{I} C_{I I}^{\mathrm{RVE}} \sqrt{2} \varepsilon_{I}=\frac{1}{|Y|^{\mathrm{RVE}}} \int_{Y} \operatorname{Tr}[\sigma \varepsilon] d Y \quad I \in[4,6] \\
& \varepsilon_{I} C_{I I}^{\mathrm{RVE}} \varepsilon_{I}+2 \varepsilon_{I} C_{I J}^{\mathrm{RVE}} \varepsilon_{J}+\varepsilon_{J} C_{J}^{\mathrm{RVE}} \varepsilon_{J} \\
& =\frac{1}{|Y|^{\mathrm{RVE}}} \int_{Y} \operatorname{Tr}[\sigma \varepsilon] d Y \\
& \varepsilon_{I} C_{I I}^{\mathrm{RVE}} \varepsilon_{I}+2 \varepsilon_{I} C_{I J}^{\mathrm{RVE}} \sqrt{2} \varepsilon_{J}+\sqrt{2} \varepsilon_{J} C_{J J}^{\mathrm{RVE}} \sqrt{2} \varepsilon_{J} \\
& =\frac{1}{|Y|^{\mathrm{RVE}}} \int_{Y} \operatorname{Tr}[\sigma \varepsilon] d Y \quad(I, J) \in[1,3] \times[4,6] \\
& \sqrt{2} \varepsilon_{I} C_{I I}^{\mathrm{RVE}} \sqrt{2} \varepsilon_{I}+2 \sqrt{2} \varepsilon_{I} C_{I J}^{\mathrm{RVE}} \sqrt{2} \varepsilon_{J}+\sqrt{2} \varepsilon_{J} C_{J J}^{\mathrm{RVE}} \sqrt{2} \varepsilon_{J} \\
& =\frac{1}{|Y|^{\mathrm{RVE}}} \int_{Y} \operatorname{Tr}[\sigma \varepsilon] d Y \quad \text { with }(I \neq J) \\
& \text { with }(I, J) \in[4,6]^{2} \text { with }(I \neq J) .
\end{aligned}
$$




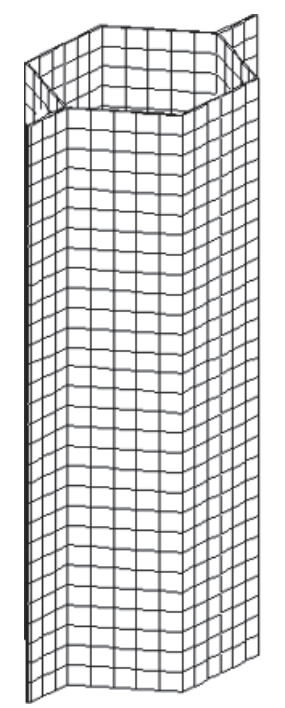

RVE honeycomb material

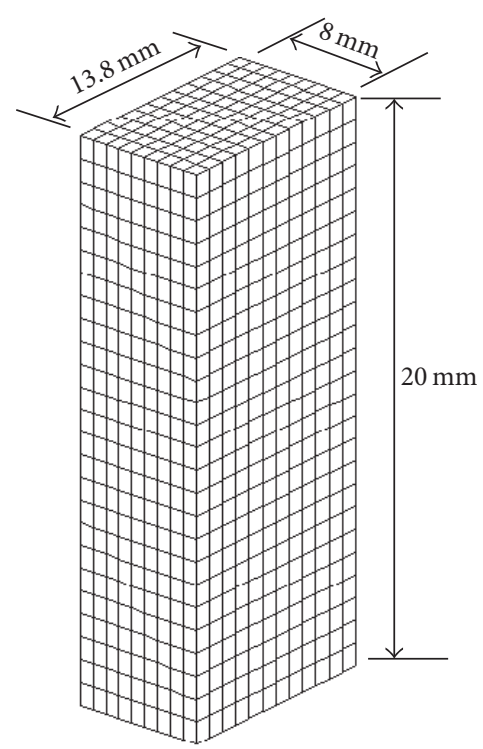

Equivalent homogenized volume

FIgURE 2: Honeycomb core RVE.

Equations (4) are used to find the components of compliance matrix $C_{I J}^{\mathrm{RVE}}$ and components of the stiffness matrix $S_{I J}^{\mathrm{RVE}}$ can be determined by using the relation $S_{I J}^{\mathrm{RVE}}=$ $\left[C_{I J}^{\mathrm{RVE}}\right]^{-1}$. The selected RVE is loaded with the imposed strain for different loading conditions corresponding to different coefficients of the compliance matrix. The resultant strain energy of the RVE can be calculated in the postprocessing after the FEA is completed. The calculated strain energy is related to the coefficients of the compliance matrix $\left(C_{I J}\right)$ by the Hill-Mandel theorem, (3). The coefficients of the compliance matrix $\left(C_{I J}\right)$ corresponding to different loading conditions are represented by (4). The orthotropic material parameters $\left(E_{11}, E_{22}, E_{33}, G_{12}, G_{13}, G_{23}, v_{12}, v_{13}\right.$, and $\left.v_{23}\right)$ can be determined from the stiffness matrix $S_{I J}^{\mathrm{RVE}}$. See the Appendix for detailed mathematical relations.

\section{3D FEA and Results}

In this section, FE analysis and results are presented and discussed in detail. Two different FE models were developed and analyzed. First, FEA of a single cell of hexagonal honeycomb representing the RVE is performed and the equivalent orthotropic properties are determined using the methodology explained in previous section. Then a sandwich beam is modeled and FEA (3PBT and 4PBT) is performed using the determined equivalent properties.

4.1. Determination of Equivalent Properties. FEA to determine the equivalent properties was done using the commercially available software CAST3M [20]. This code was developed by the "Département Mécanique et Technologie (DMT) du Commissariat français à l'Energie Atomique (CEA), France." The purpose of the development of CAST3M is to provide high level support for the design and analysis of structures and components in the research field. In this context, CAST3M not only incorporates the process of calculations but also serves as preprocessor for the modeling of structural parts and analysis of the final results (posttreatment).

The honeycomb core is modeled with three-dimensional brick elements having 20 nodes (CU20) [20]. The core material is polypropylene with a density of $80.0 \mathrm{Kg} / \mathrm{m}^{3}$ and a sheet thickness of $0.24 \mathrm{~mm}$. The unit cell is $8.0 \mathrm{~mm}$ wide and $20 \mathrm{~mm}$ high (see Figure 1).

As adopted by Gornet et al. [13, 15], FEA was done for different loading conditions to determine the orthotropic coefficients of the compliance matrix. The principal orthotropic directions are shown in Figure 1. The boundary conditions for five different loadings are shown in Figure 3. Von Mises stress profiles of $20 \mathrm{~mm}$ thick core for three tensile loading conditions are shown in Figure 4. The determined orthotropic properties and their comparison with analytical results as proposed by Gibson and Ashby [8] and Chamis et al. [6] are given in Table 1 .

Honeycomb cores are usually designed for out-of-plane normal (E33) and shear (G13, G23) loading conditions. Gibson and Ashby [8] presented the solution for orthotropic properties of honeycomb cores; however, they did not consider the effects of double wall thickness and periodic nature of core media. Due to this reason, the values for E11 and E22 are not only similar to each other but also lower than those depicted by FE results. Likewise, the values of G13 and G23 are also identical for the same reasons. The out-of-plane modulus predicted by the FEA is in close approximation to the Gibson and Ashby results. Still the value of E33 is towards the lower side for the Ashby and Gibson due to constant wall approximation. Chamis et al. [6] also proposed approximated formulas to calculate the equivalent orthotropic properties of honeycomb structures. 

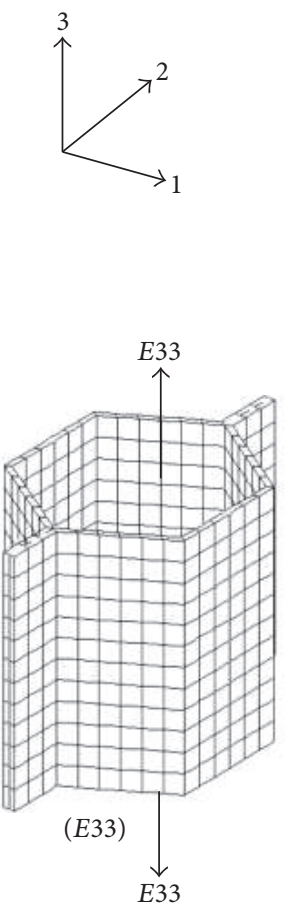

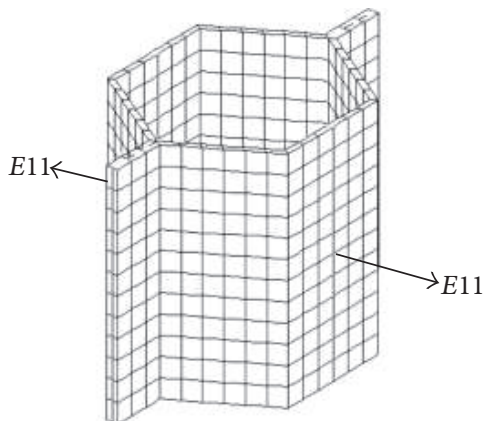

$(E 11)$

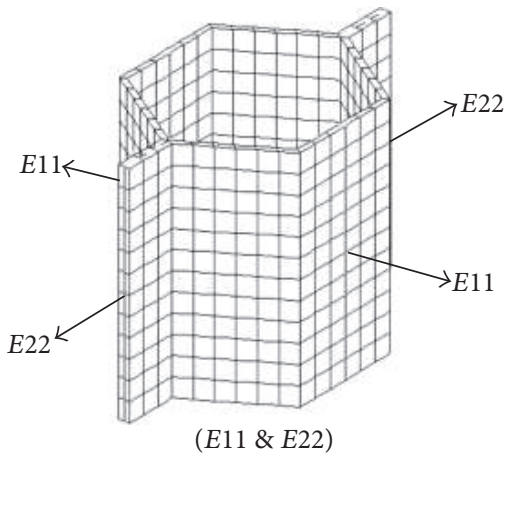

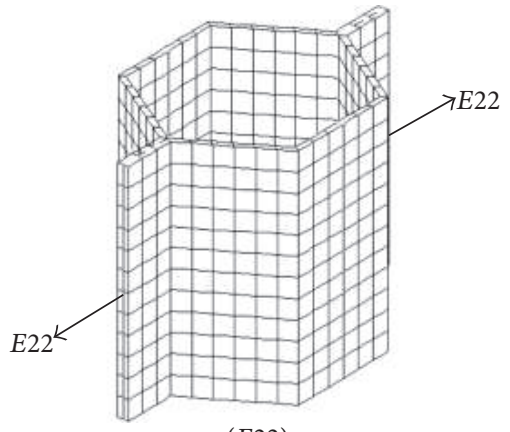

$(E 22)$

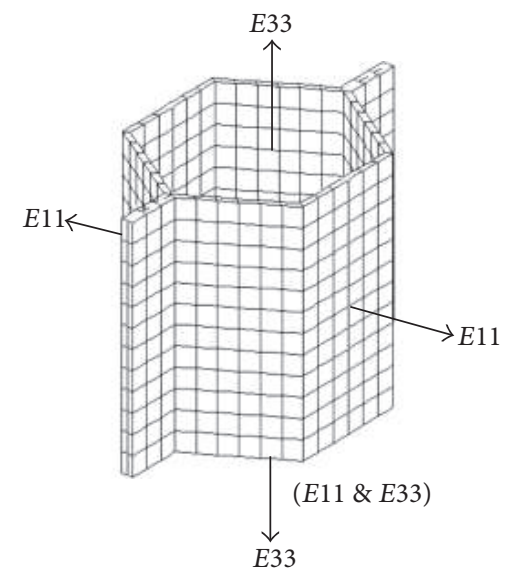

FIGURE 3: Boundary conditions for five different loadings.

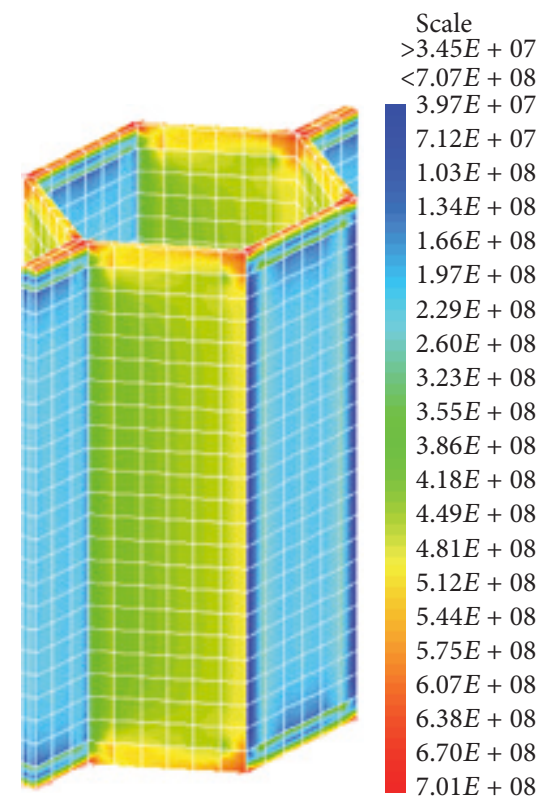

E11

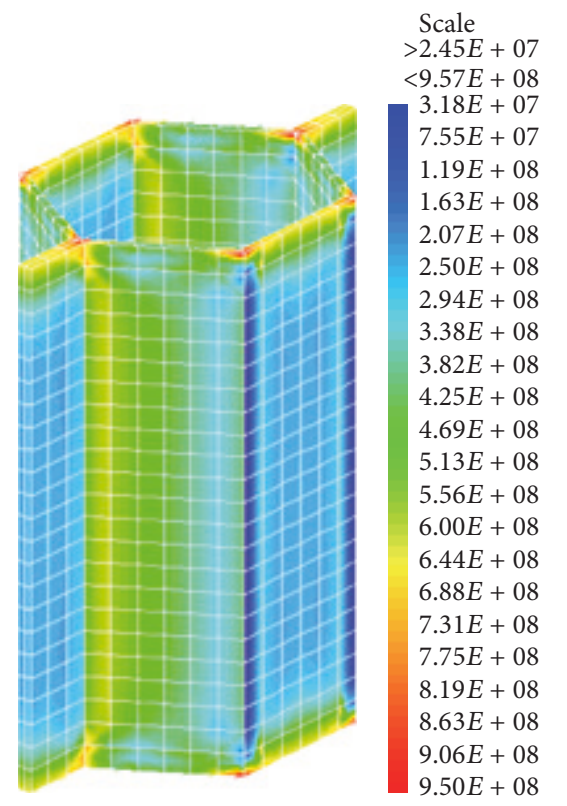

E22

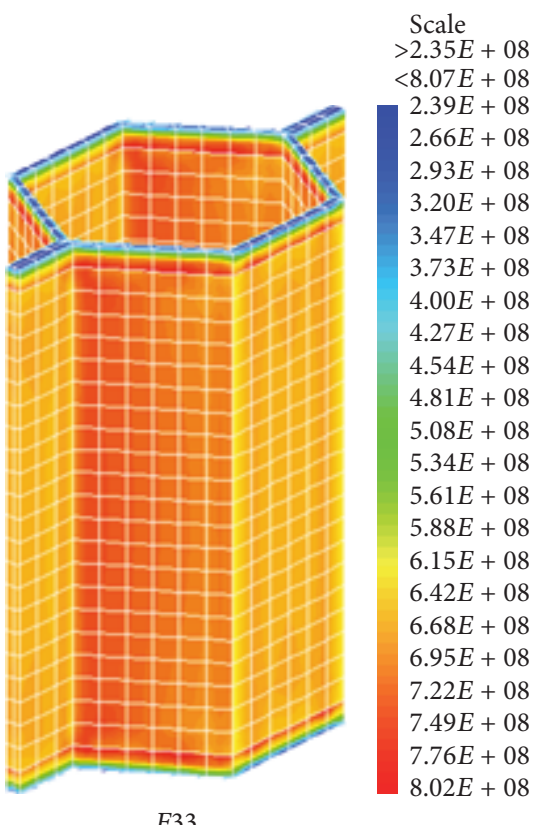

E33

FIgURE 4: Von Mises stress profile for different loading conditions on hexagonal core. 
TABLE 1: Comparison of FEA results with published $[6,8]$ data.

\begin{tabular}{lccc}
\hline Property & Gibson and Ashby [8] \\
Unit cell & $6.9 E 6$ & Chamis et al. [6] \\
$E_{11}\left(\mathrm{~N} / \mathrm{m}^{2}\right)$ & $10.2 E 7$ & $4.2 E 5$ & $7.8 E 7$ \\
$E_{22}\left(\mathrm{~N} / \mathrm{m}^{2}\right)$ & $1.97 E 6$ & $1.05 E 5$ & $1.85 E 7$ \\
$E_{33}\left(\mathrm{~N} / \mathrm{m}^{2}\right)$ & $1.44 E 7$ & $1.37 E 7$ & $1.03 E 7$ \\
$G_{12}\left(\mathrm{~N} / \mathrm{m}^{2}\right)$ & $2.2 E 7$ & $1.37 E 7$ & $1.37 E 7$ \\
$G_{13}\left(\mathrm{~N} / \mathrm{m}^{2}\right)$ & 0.81 & 0.99 & $2.06 E 7$ \\
$G_{23}\left(\mathrm{~N} / \mathrm{m}^{2}\right)$ & 0.024 & 0.031 & 0.42 \\
$v_{12}$ & 0.028 & 0.0054 & 0.42 \\
$v_{13}$ & & 0.42 \\
$v_{23}$ & &
\end{tabular}

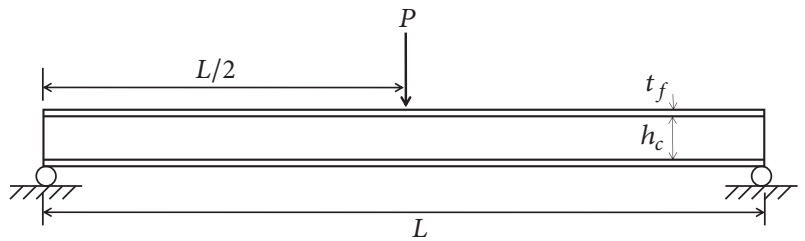

FIGURE 5: Three-point bend test (3PBT) specimen.

The formulations proposed by Chamis et al. overestimated the calculated values. Readers may consult the work of Gibson and Ashby and Chamis et al. for more details $[6,8]$.

In the present work, only one honeycomb core cell with actual double wall thickness is modeled and results are obtained by applying periodic displacement boundary conditions. The predicted out-of-plane elastic modulus E33 and the two shear moduli G13 and G23 were found in good agreement with the values obtained by Chamis et al. [6]. The identified equivalent properties were used for the 3D 4PBT and 3PBT FE analyses in the next section.

4.2. 3D FE Analysis of Three- and Four-Point Bend Tests. The sandwich structure usually consists of central core materials covered by the face sheets on both sides of the core. In the present study, two different types of analyses were performed, where the face sheets were modeled with the shell elements in the first and with the 3D solid elements in the second analysis. However, the core material is modeled by $3 \mathrm{D}$ solid elements in both the cases. The authenticity of the performed analysis has been established by comparing the FE results with the reported work $[2,21]$.

The geometry and boundary conditions for 3PBT and 4PBT specimens are shown in Figures 5 and 6, respectively. Considering the symmetry of 4PBT, only half of the specimen is modeled and analysis is performed by employing symmetric boundary conditions. The specimen length is $L$ $=300 \mathrm{~mm}$, the face sheet thickness is $t_{f}=1 \mathrm{~mm}$, and the width is $b=35 \mathrm{~mm}$ for all the specimens. A core height $(h c)$ of $20 \mathrm{~mm}$ is used for both 3PBT and 4PBT analyses. The 3D
FE analyses are performed using the commercial software Abaqus/Standard. C3D8R solid elements are used for core material modeling. S4R shell and C3D8R solid elements are used for face sheet material modeling.

Figures 7 and 8 present the FE results of force versus deflection curve for $3 \mathrm{PBT}$ and $4 \mathrm{PBT}$, respectively. Note that changing the type of elements from solid to shell did not reflect any noticeable difference in the FE results. Nevertheless, the simulation results are in close agreement with the experimental ones.

The above simulation results on 3PBT and 4PBT indicate that the adopted strategy to model the hexagonal honeycomb material by equivalent orthotropic material gives good results. Hence, instead of modeling a large number of hexagonal cells, one can determine the equivalent properties by modeling a single hexagonal cell as an RVE only.

Figures 9 and 10 present the Von Mises and normal stress $\left(\sigma_{33}\right)$ profile on the 3PBT specimen for both core and face sheet materials, respectively.

Similarly Figures 11 and 12 show the Von Mises and normal stress $\left(\sigma_{33}\right)$ profile on the $4 \mathrm{PBT}$ specimen for core and face sheet materials, respectively. The normal stress $\left(\sigma_{33}\right)$ distribution/trend along a specific path of core material for both 3PBT and 4PBT is discussed here. The selected nodal paths along the height and width of the core are shown in Figure 13 for 3PBT and in Figure 14 for 4PBT. The stress distribution is studied at the nodes along width direction, where the loading is imposed and the same path is extended along the height for the creation of second set of nodes.

Figure 15 presents the variation of normal stress $\left(\sigma_{33}\right)$ along the core height, starting from the top to the bottom node for 3PBT (Figure 13). The normal stress produced is compressive in nature because of the direction of applied load at the selected nodes. The top node stress value is observed maximum and it decreases gradually along the path towards the bottom node. Figure 16 shows the variation of normal compressive stress along the width for 3PBT. The stress value increased as it moves from corner node and then 


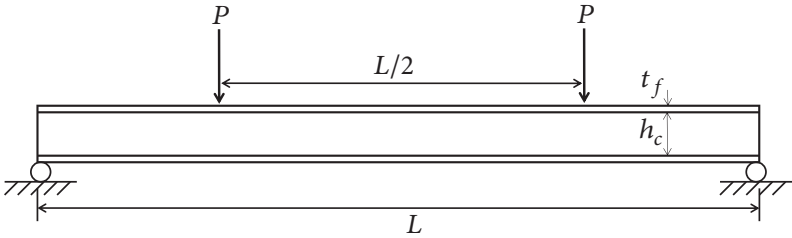

(a)

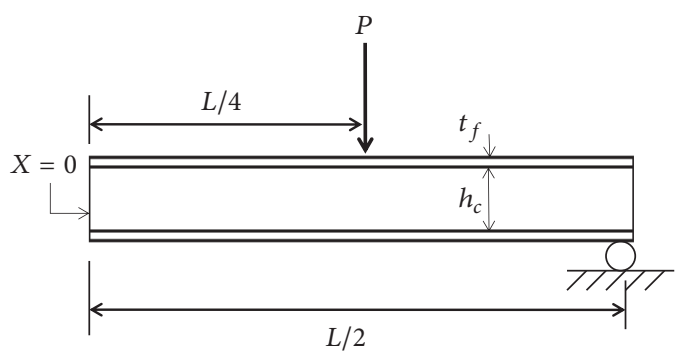

(b)

FIGURE 6: Four-point bend test (4PBT) specimen: (a) full length; (b) symmetric.

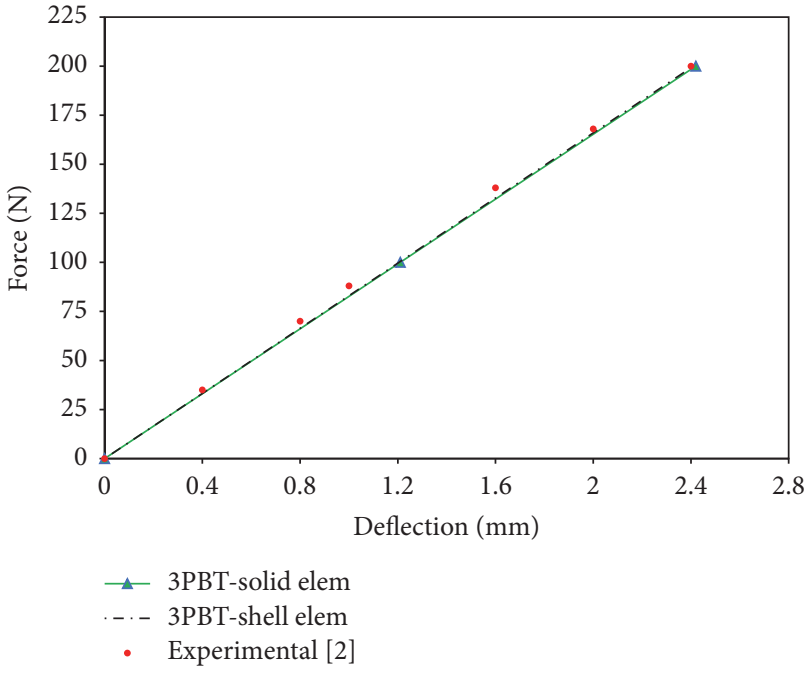

FIgURE 7: Force versus deflection curve (3PBT).

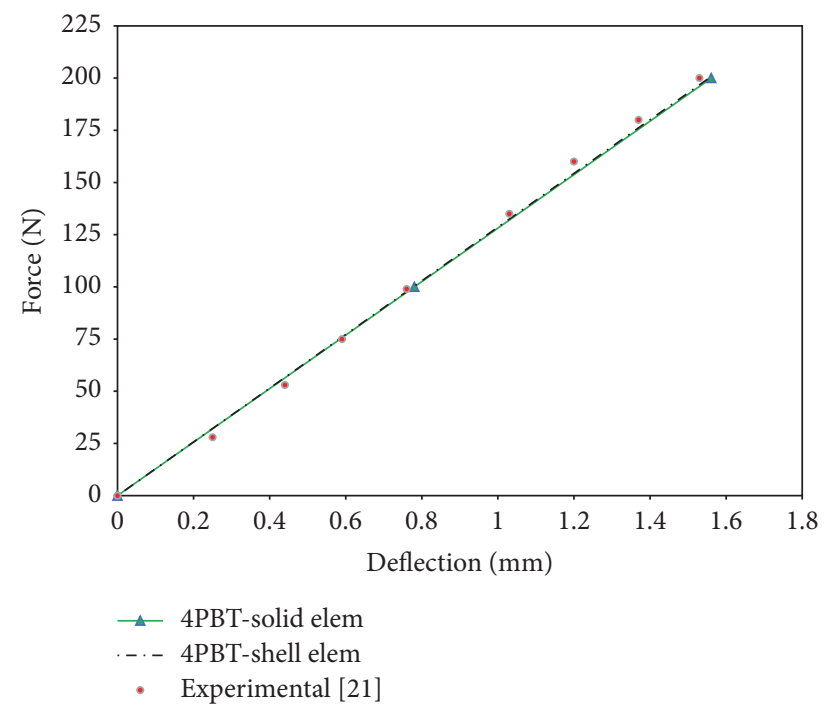

Figure 8: Force versus deflection curve (4PBT).

tends to stabilize as it reaches the middle node; after reaching the middle node the exact symmetry is observed for the remaining half width.

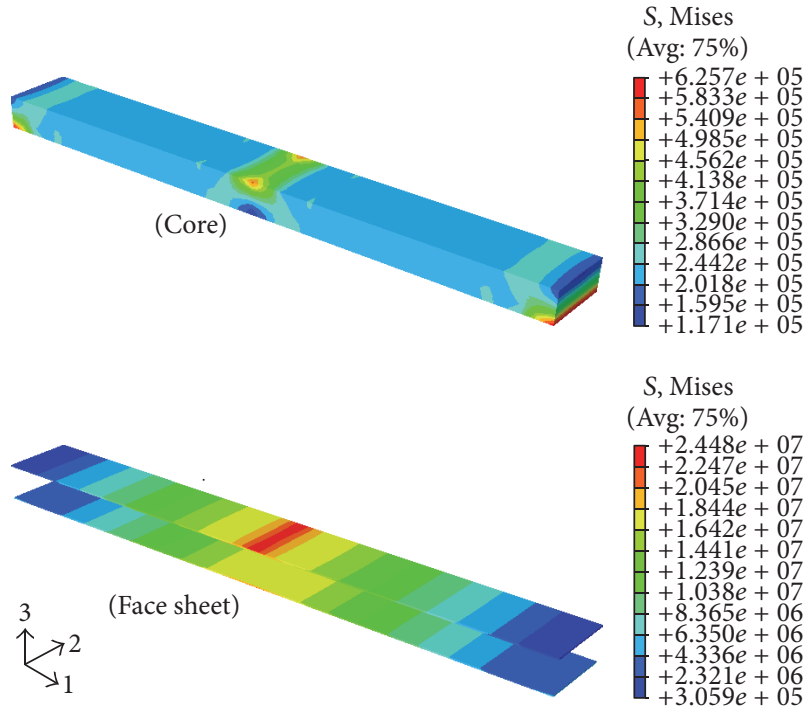

FIgUre 9: Von Mises stress profile (3PBT).

Similarly, Figures 17 and 18 present the normal compressive stress variation along the selected nodal paths for height and width directions for 4PBT. Although the stress magnitudes observed in the 4PBT are lesser as compared to those of 3PBT, the trends are quite similar for both the cases. The lower values of normal stress for $4 \mathrm{PBT}$ can be attributed to the small bending arm's length for the given loading in comparison to 3PBT; see Figures 5 and 6.

\section{Conclusion}

In this article, the strain energy based homogenization methodology is explained to determine the equivalent orthotropic properties of honeycomb sandwich structures. The obtained properties are further successfully employed to simulate the 3PBT and 4PBT. A 3D representative volume element (RVE) of one cell of honeycomb is selected to apply the homogenization technique rather than selecting a continuous structure having multiple cells, hence considerably reducing the computational time and effort. In the first part, homogenization is performed for honeycomb core material and determined orthotropic properties are compared with the properties based on available theories of different authors. 


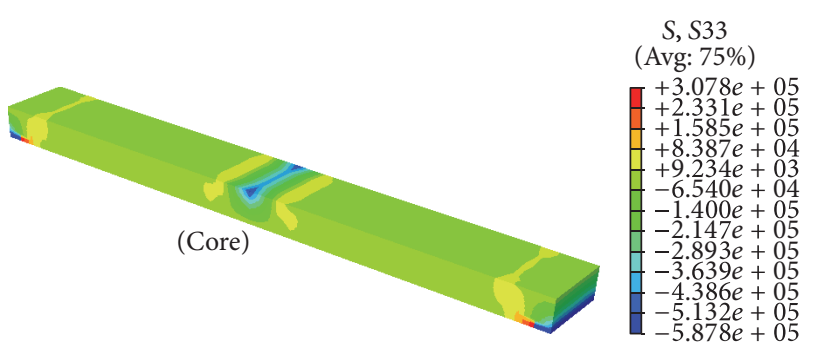

$S, S 33$

(Avg: $75 \%$ )

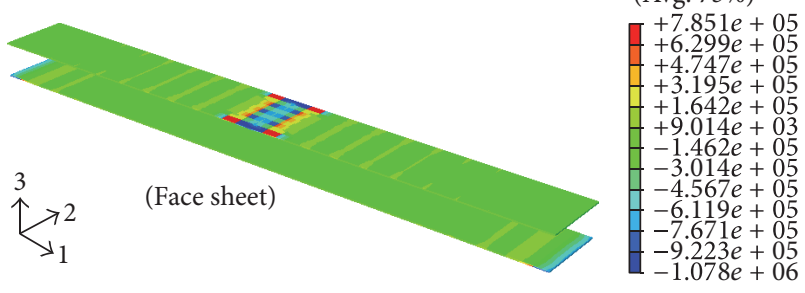

FIGURE 10: Normal stress $\left(\sigma_{33}\right)$ profile (3PBT).

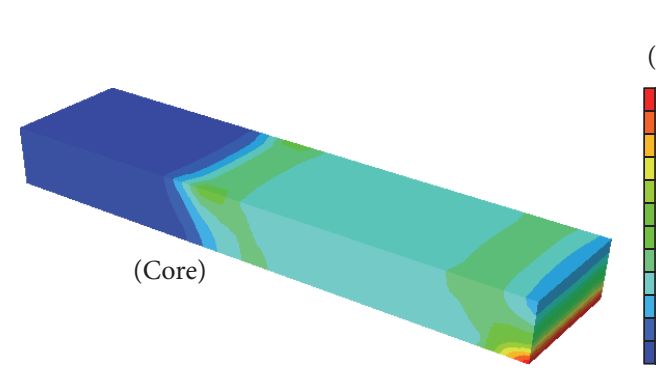

S, Mises (Avg: 75\%) $\begin{aligned} & +7.379 e+05 \\ & +6.766 e+05 \\ & +6.153 e+05 \\ & +5.539 e+05 \\ & +4.926 e+05 \\ & +4.312 e+05 \\ & +3.699 e+05 \\ & +3.086 e+05 \\ & +2.472 e+05 \\ & +1.859 e+05 \\ & +1.246 e+05 \\ & +6.322 e+04 \\ & +1.877 e+03\end{aligned}$

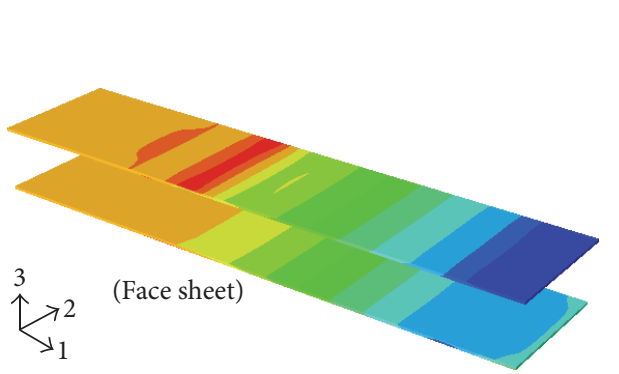

$S$, Mises (Avg: 75\%)

$+1.502 e+07$ $+1.379 e+07$ $+1.256 e+07$ $+1.133 e+07$ $+1.011 e+07$ $+8.877 e+06$ $+7.648 e+06$ $+6.420 e+06$ $+5.191 e+06$ $+3.962 e+06$ $+2.733 e+06$ $+1.505 e+06$ Figure 11: Von Mises stress profile (4PBT).

In the second part, 3D FEA of 3PBT and 4PBT are performed based on the determined equivalent orthotropic properties. The bend test results for both analyses are compared with experimental data and are found in good agreement.

\section{Appendix}

The constitutive equations representing the $3 \mathrm{D}$ stress-strain relation in terms of $6 \times 6$ compliance matrix, $C_{I J}$ (Herakovich, 1997) [17], are

$$
\sigma_{1}=C_{I J} \varepsilon_{J}
$$

$S, S 33$

(Avg: 75\%)

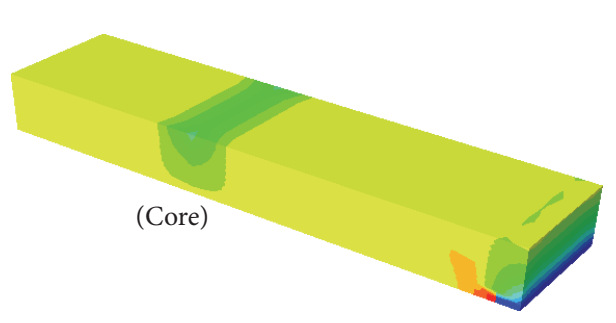

$+3.047 e+05$

$+2.204 e+05$

$+1.361 e+05$

$+5.181 e+04$

$-3.249 e+04$
$-1.168 e+05$

$-1.168 e+05$

$-2.854 e+05$

$-3.697 e+05$

$-4.540 e+05$

$-5.383 e+05$

$-6.226 e+05$

$-7.069 e+05$

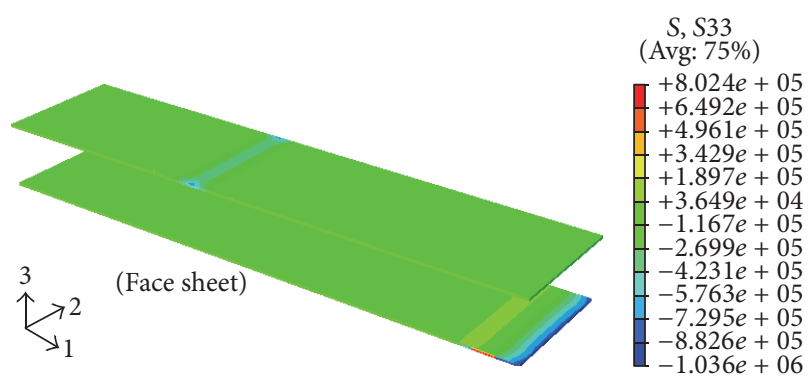

FIgURE 12: Normal stress $\left(\sigma_{33}\right)$ profile (4PBT).

$\left[\begin{array}{c}\sigma_{11} \\ \sigma_{22} \\ \sigma_{33} \\ \sqrt{2} \sigma_{23} \\ \sqrt{2} \sigma_{13} \\ \sqrt{2} \sigma_{12}\end{array}\right]$

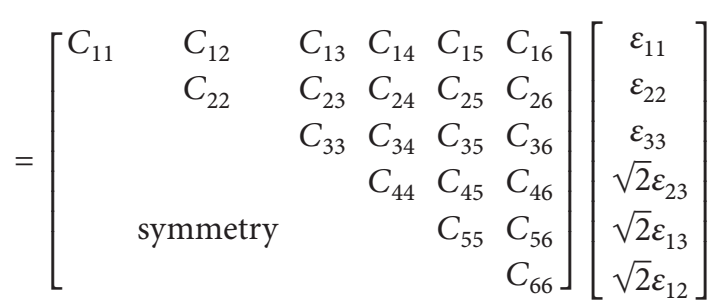

The inverse relationship in terms of $6 \times 6$ stiffness matrix, $S_{I J}$, may be expressed as follows (Herakovich, 1997) [17]:

$$
\begin{gathered}
\varepsilon_{1}=S_{I J} \sigma_{J} \\
{\left[\begin{array}{c}
\varepsilon_{11} \\
\varepsilon_{22} \\
\varepsilon_{33} \\
\sqrt{2} \varepsilon_{23} \\
\sqrt{2} \varepsilon_{13} \\
\sqrt{2} \varepsilon_{12}
\end{array}\right]}
\end{gathered}
$$

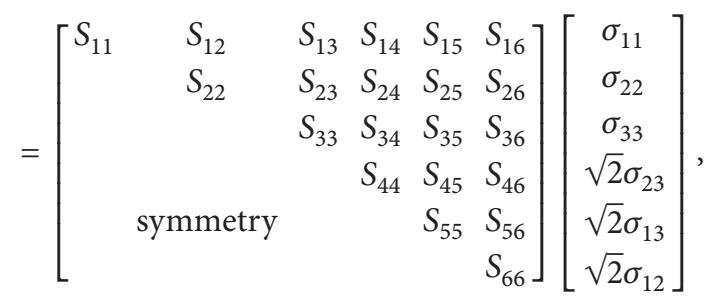

where $S_{I J}=C_{I J}^{-1}$. 


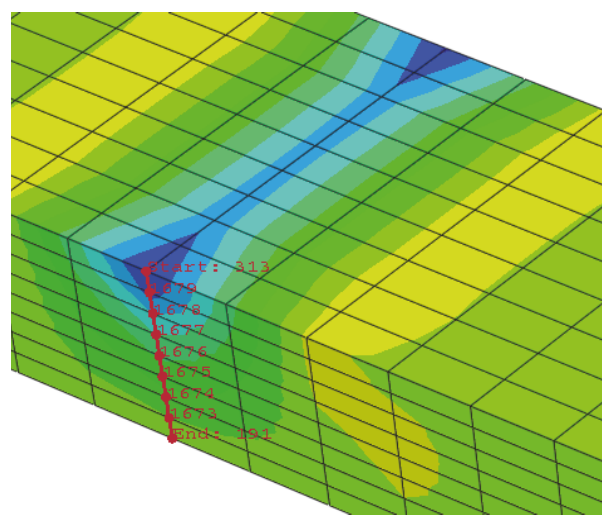

(a)

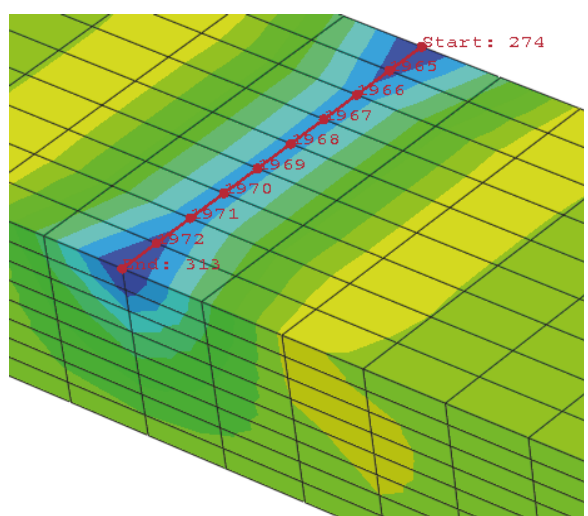

(b)

FIGURE 13: Selected nodal path direction for 3PBT: (a) height; (b) width.

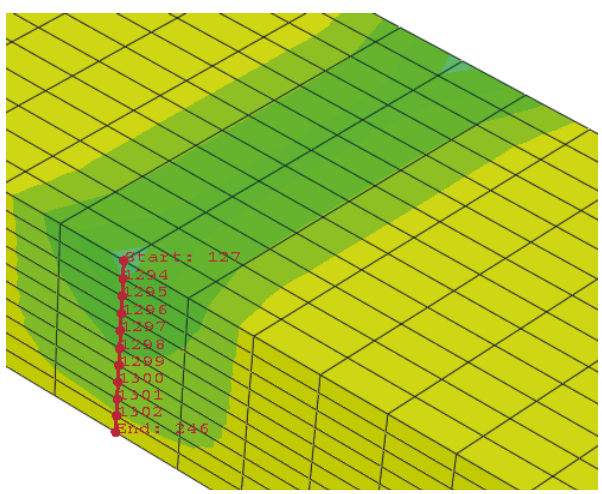

(a)

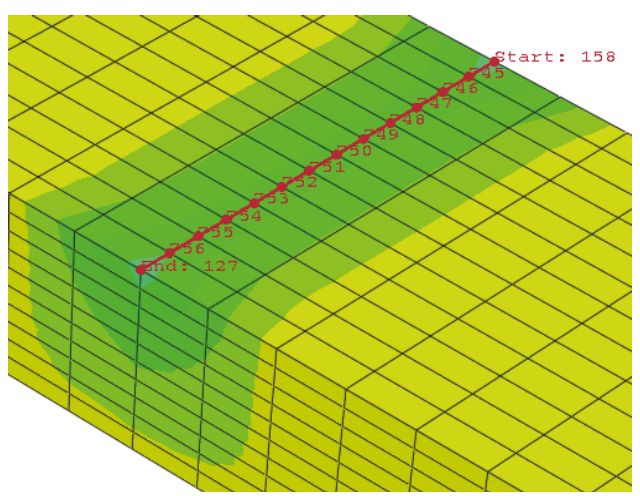

(b)

FIGURE 14: Selected nodal path direction for 4PBT: (a) height; (b) width.

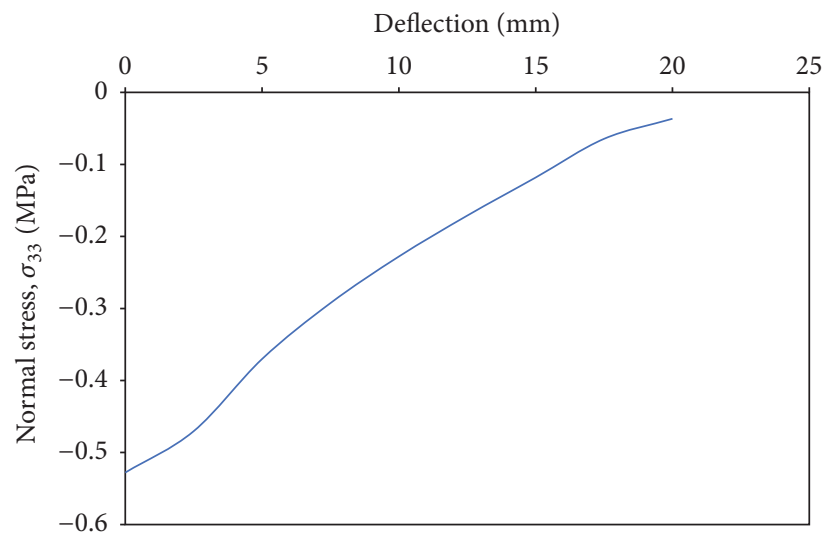

FIgURE 15: Normal stress distribution along the height direction (3PBT). 


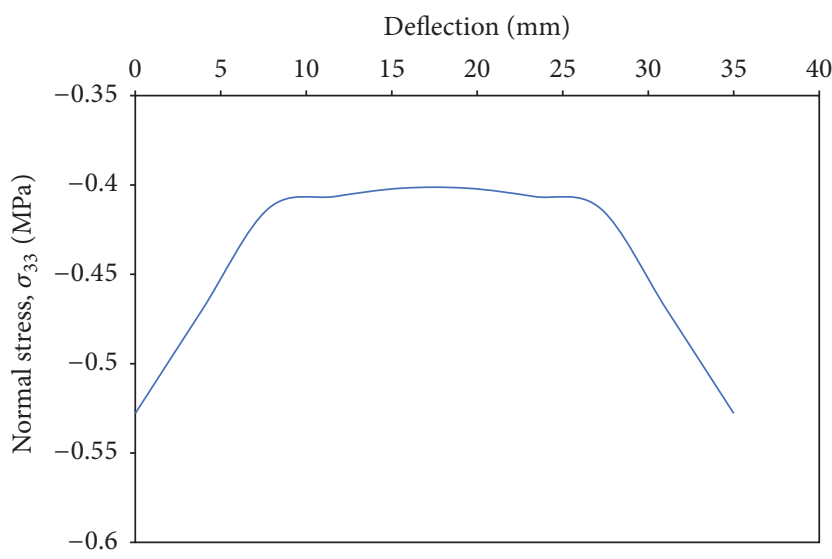

FIGURE 16: Normal stress distribution along the width direction (3PBT).

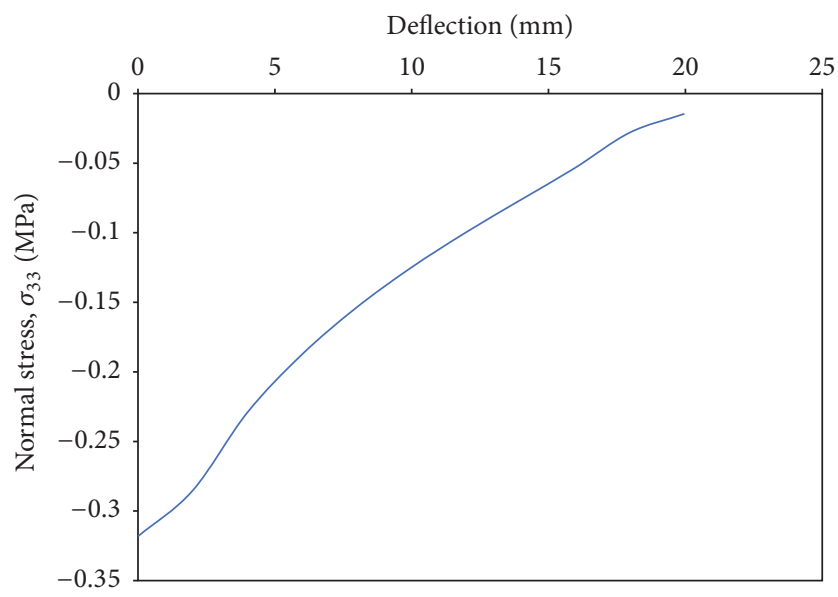

Figure 17: Normal stress distribution along the height direction (4PBT).

The Hill-Mandel theorem equates the RVE energy to the average of the energies of its components:

$$
\varepsilon_{I} C_{I J} \varepsilon_{J}=\frac{1}{|Y|} \int_{Y} \operatorname{Tr}[\sigma \varepsilon] d Y .
$$

Components of the $6 \times 6$ compliance matrix, $C_{I J}$, are given in (A.2). The coefficients of the compliance matrix are calculated using (4) for different imposed strain loading conditions. The components of the stiffness matrix may be calculated using relation $S_{I J}=C_{I J}{ }^{-1}$. Stiffness matrix for the orthotropic material is represented by (A.5) where some coefficients become zero due to symmetry of three mutually orthogonal planes (Herakovich, 1997) [17].

$$
\left[\begin{array}{cccccc}
S_{11} & S_{12} & S_{13} & 0 & 0 & 0 \\
S_{12} & S_{22} & S_{23} & 0 & 0 & 0 \\
S_{13} & S_{23} & S_{33} & 0 & 0 & 0 \\
0 & 0 & 0 & S_{44} & 0 & 0 \\
0 & 0 & 0 & 0 & S_{55} & 0 \\
0 & 0 & 0 & 0 & 0 & S_{66}
\end{array}\right] .
$$

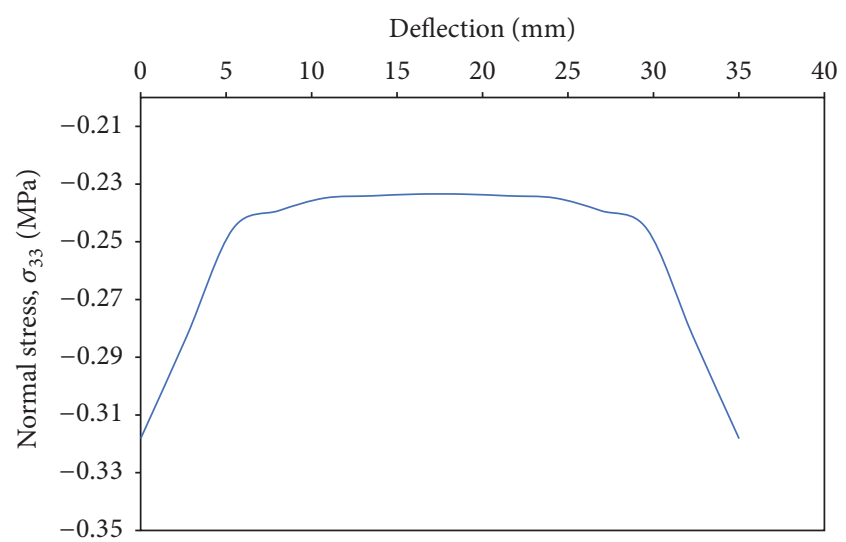

FIGURE 18: Normal stress distribution along the width direction (4PBT).

The orthotropic material parameters $\left(E_{11}, E_{22}, E_{33}, G_{12}\right.$, $G_{13}, G_{23}, v_{12}, v_{13}$, and $\left.v_{23}\right)$ can be determined from stiffness matrix $S_{I J}$; see (A.6) (Herakovich, 1997) [17].

$$
\begin{aligned}
& {\left[\begin{array}{cccccc}
S_{11} & S_{12} & S_{13} & 0 & 0 & 0 \\
S_{12} & S_{22} & S_{23} & 0 & 0 & 0 \\
S_{13} & S_{23} & S_{33} & 0 & 0 & 0 \\
0 & 0 & 0 & S_{44} & 0 & 0 \\
0 & 0 & 0 & 0 & S_{55} & 0 \\
0 & 0 & 0 & 0 & 0 & S_{66}
\end{array}\right]} \\
& =\left[\begin{array}{ccccccc}
\frac{1}{E_{1}} & \frac{-v_{12}}{E_{1}} & \frac{-v_{13}}{E_{1}} & 0 & 0 & 0 \\
\frac{-v_{21}}{E_{2}} & \frac{1}{E_{2}} & \frac{-v_{23}}{E_{2}} & 0 & 0 & 0 \\
\frac{-v_{31}}{E_{3}} & \frac{-v_{32}}{E_{3}} & \frac{1}{E_{3}} & 0 & 0 & 0 \\
0 & 0 & 0 & \frac{1}{2 G_{23}} & 0 & 0 \\
0 & 0 & 0 & 0 & \frac{1}{2 G_{13}} & 0 \\
0 & 0 & 0 & 0 & 0 & \frac{1}{2 G_{12}}
\end{array}\right] .
\end{aligned}
$$

\section{Conflicts of Interest}

The authors declare that there are no conflicts of interest regarding the publication of this paper.

\section{References}

[1] C. Xu, H. Yanjiao, W. Shou, L. Chun, and F. Luping, "Stress distribution on sandwich structure with triangular grid cores suffered from bending load," International Journal of Aerospace Engineering, vol. 2015, Article ID 723487, 8 pages, 2015.

[2] J. Arbaoui, Y. Schmitt, J. L. Pierrot, and F. X. Royer, "Numerical simulation and experimental bending behaviour of multilayer sandwich structures," Journal of Theoretical and Applied Mechanics, vol. 52, no. 2, pp. 431-442, 2014. 
[3] A. Zinno, A. Prota, E. Di Maio, and C. E. Bakis, "Experimental characterization of phenolic-impregnated honeycomb sandwich structures for transportation vehicles," Composite Structures, vol. 93, no. 11, pp. 2910-2924, 2011.

[4] B. P. Russell, T. Liu, N. A. Fleck, and V. S. Deshpande, "Quasistatic three-point bending of carbon fiber sandwich beams with square honeycomb cores," Journal of Applied Mechanics, Transactions ASME, vol. 78, no. 3, Article ID 031008, 2011.

[5] M. Giglio, A. Gilioli, and A. Manes, "Numerical investigation of a three point bending test on sandwich panels with aluminum skins and Nomex ${ }^{\mathrm{TM}}$ honeycomb core," Computational Materials Science, vol. 56, pp. 69-78, 2012.

[6] C. Chamis, R. Aiello, and P. Murthy, "Fiber composite sandwich thermostructural behavior: computational simulation. Structures," in Proceeding of the Structural Dynamics And Materials Conference, pp. 370-381, American Institute of Aeronautics and Astronautics, San Antonio, TX, USA, 1986.

[7] S. Kelsey, R. A. Gellatly, and B. W. Clark, "The shear modulus of foil honeycomb cores: a theoretical and experimental investigation on cores used in sandwich construction," Aircraft Engineering and Aerospace Technology, vol. 30, no. 10, pp. 294302, 1958 .

[8] L. J. Gibson and M. F. Ashby, Cellular Solids: Structures and Properties, Cambridge University Press, Cambridge, UK, 2nd edition, 1997.

[9] J. Zhang and M. F. Ashby, "The out-of-plane properties of honeycombs," International Journal of Mechanical Sciences, vol. 34, no. 6, pp. 475-489, 1992.

[10] J. W. Klintworth and W. J. Stronge, "Elasto-plastic yield limits and deformation laws for transversely crushed honeycombs," International Journal of Mechanical Sciences, vol. 30, no. 3-4, pp. 273-292, 1988.

[11] J. Hohe and W. Becker, "Effective stress-strain relations for twodimensional cellular sandwich cores: homogenization, material models, and properties," Applied Mechanics Reviews, vol. 55, no. 1, pp. 61-87, 2002.

[12] Q. S. Yang and W. Becker, "A comparative investigation of different homogenization methods for prediction of the macroscopic properties of composites," Computer Modeling in Engineering and Sciences, vol. 6, no. 4, pp. 319-332, 2004.

[13] L. Gornet, G. Marckmann, and M. Lombard, "Failure and elastic properties of Nomex ${ }^{\circledast}$ honeycombs: periodic homogeneization and mechanical simulation," Mecanique et Industries, vol. 6, no. 6, pp. 595-604, 2005.

[14] Y. Zhang and Z. Xia, "Micromechanical analysis of interface damage for fiber reinforced composite laminates," Computers Materials \& Continua, vol. 38, pp. 3689-3717, 2005.

[15] L. Gornet, S. Marguet, and G. Marckmann, "Numerical modelling of Nomex ${ }^{\circledR}$ honeycomb cores: failure and effective elastic properties," in Proceeding of the III European Conference on Computational Mechanics, 509, vol. 509, 2006.

[16] H. Ijaz, M. Asad, A. Memon, KB. Ahmed, H. Abbasi, and AN. Laghari, "Strain energy based homogenization method to find the equivalent orthotropic properties of sandwich structures," Sindh University Research Journal (Science Series), vol. 46, no. 1, pp. 93-98, 2014.

[17] C. T. Herakovich, Mechanics of Fibrous Composites, John Wiley and Sons Ltd, New York, NY, USA, 1st edition, 1997.

[18] X. Li, Q. Liu, and J. Zhang, "A micro-macro homogenization approach for discrete particle assembly-Cosserat continuum modeling of granular materials," International Journal of Solids and Structures, vol. 47, no. 2, pp. 291-303, 2010.
[19] S. Forest, "Mechanics of generalized continua: construction by homogenizaton," Journal de Physique IV, vol. 18, pp. 39-48, 1998.

[20] P. Verpeaux, T. Charras, and A. Millard, "Castem 2000: Une approche moderne du calcul des structures," in Calcul des Structures et Intelligence Artificielle, J. M. Fouet, P. Ladevèze, and R. Ohayon, Eds., vol. 2, pp. 227-261, 1988.

[21] J. Arbaoui, Y. Schmitt, J.-L. Pierrot, and F.-X. Royer, "Effect of core thickness and intermediate layers on mechanical properties of polypropylene honeycomb multi-layer sandwich structures," Archives of Metallurgy and Materials, vol. 59, no. 1, pp. 11-16, 2014. 

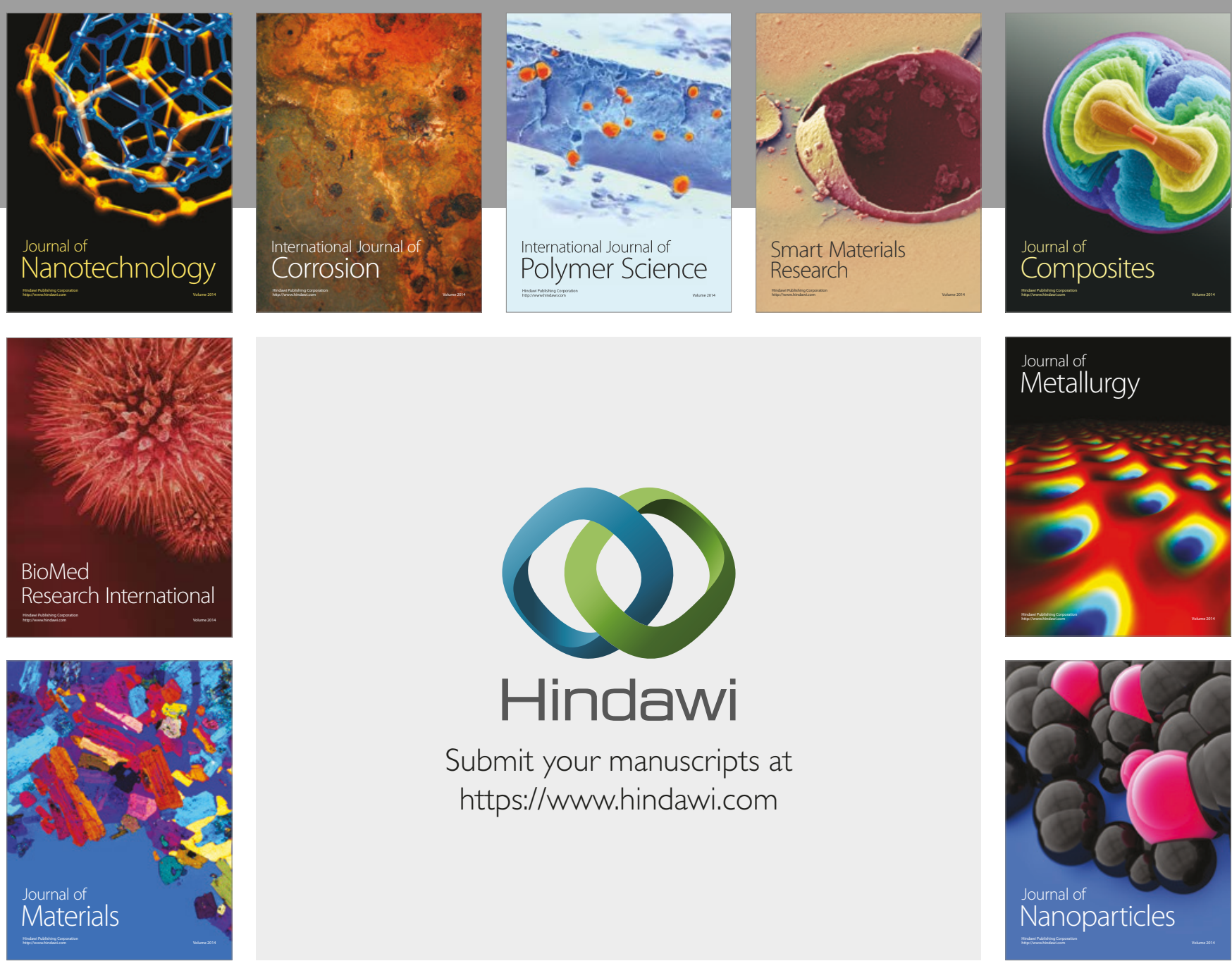

\section{Hindawi}

Submit your manuscripts at

https://www.hindawi.com
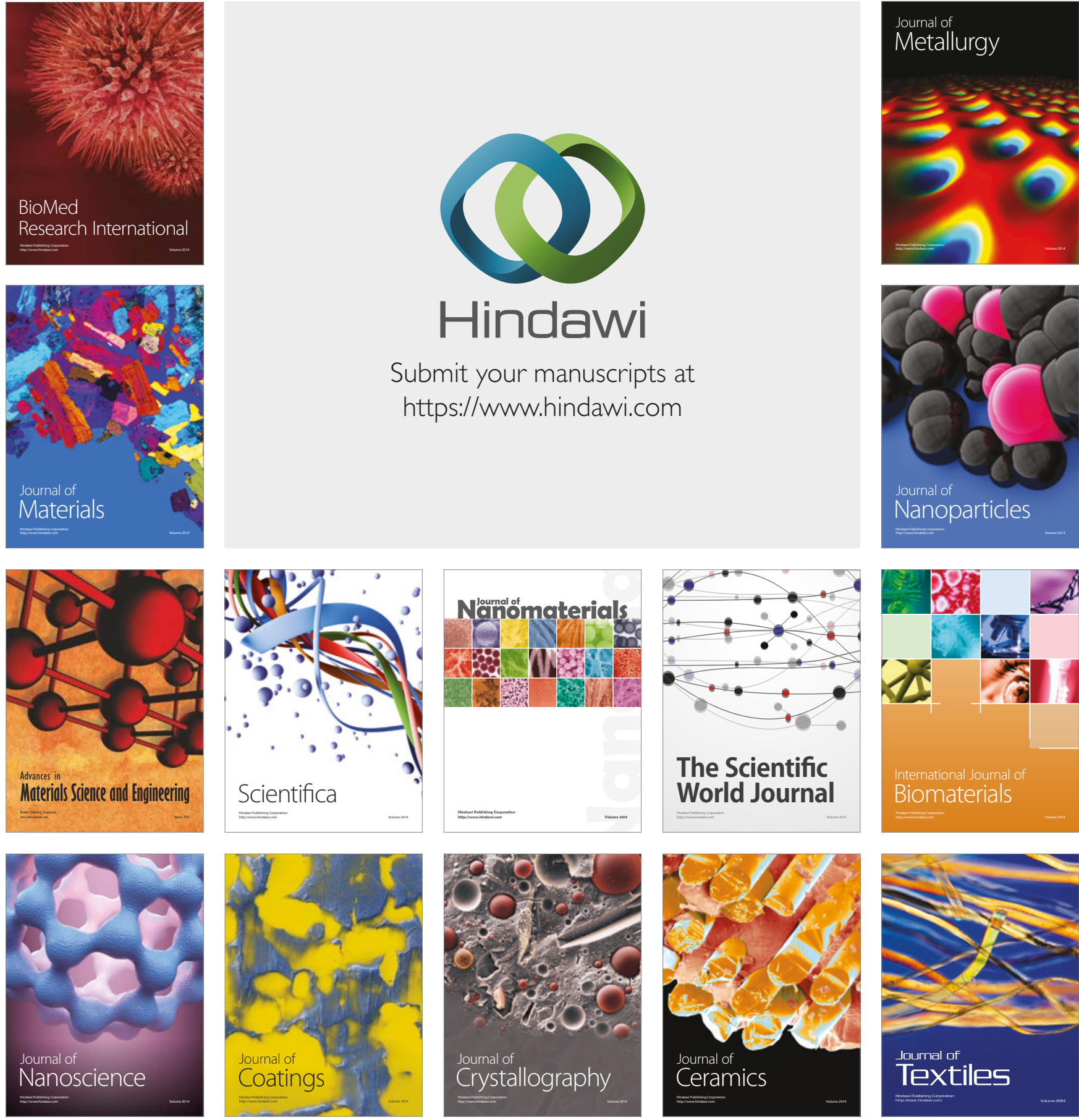

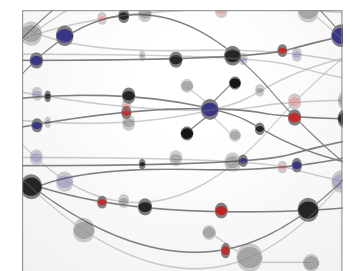

The Scientific World Journal
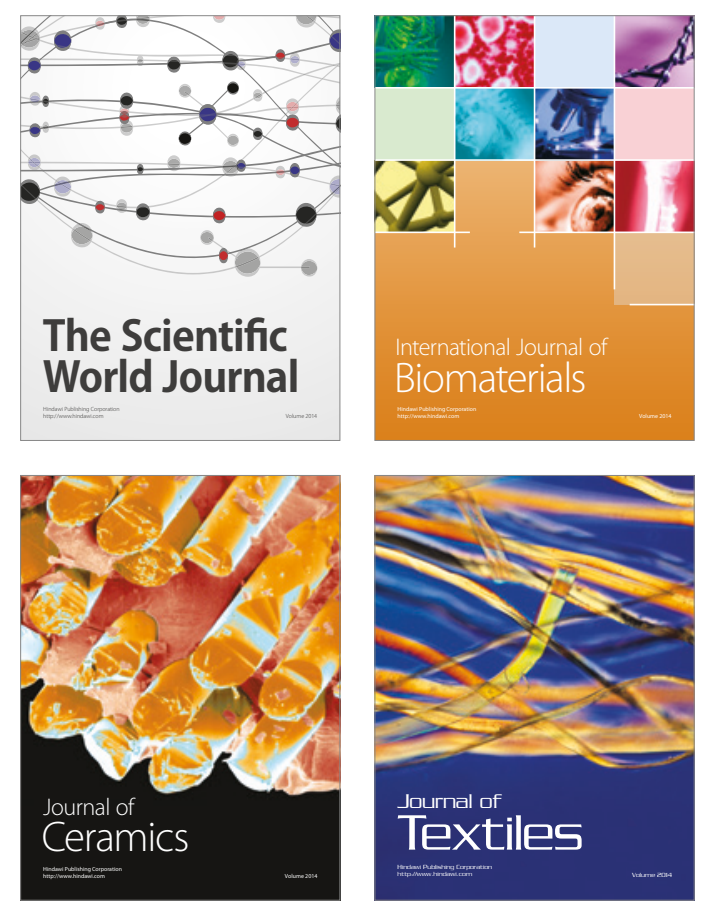